\title{
The Expression Level of Neutrophil CD64 Is a Useful Marker of Systemic Inflammation Associated with HIV Infection
}

\author{
Fujiko Mitsumoto-Kaseida, Masayuki Murata, Kazuya Ura, Koji Takayama, Satoshi Hiramine, \\ Motohiro Shimizu, Kazuhiro Toyoda, Eiichi Ogawa, and Norihiro Furusyo
}

\begin{abstract}
CD64 is an Fc-gamma-receptor type 1. The expression level of neutrophil CD64 (nCD64) is a known bacterial infection marker, and it also increases in viral infections. We examined the absolute nCD64 before and after the initiation of antiretroviral therapy (ART) to determine its role as an infection and inflammation marker of human immunodeficiency virus (HIV) infection. In this prospective observational study, 94 HIV-infected patients were enrolled and classified into ART $(n=62)$, ART naive $(n=24)$, and acute/early phase groups $(n=8)$. The median nCD64 was 1,430 molecules/cell in the ART group, 2,994 in the ART naive group, 4,625 in the acute/early phase group, and 1,196 in the healthy control group. The nCD64 in the ART group was significantly higher compared with the healthy controls $(p=.041)$, and the nCD64 in the ART naive and acute/early phase groups was significantly higher compared with the ART group (both $p<.001$ ). In the ART naive group, nCD64 was significantly higher in patients with than without concomitant infections $(3,942 \pm 1,519$ vs. $2,300 \pm 784, p=.004)$. However, this was influenced by the fact that nCD64 elevated as the stage of HIV infection progressed. nCD64 decreased significantly during the 24 weeks after starting ART ( $p=.004)$, although an upward trend in nCD64 was observed at weeks 2 and 4, without symptoms. When immune reconstitution inflammatory syndrome occurred, nCD64 elevated with a wider range than did C-reactive protein. This preliminary study suggests that nCD64 would be useful as a marker of the systemic inflammation of HIV-infected patients.
\end{abstract}

Keywords: HIV, immune activation, antiretroviral therapy, inflammation

\section{Introduction}

C D64, AN FC-GAMMA-RECEPTOR type 1 (Fc $\gamma \mathrm{RI})$, is a membrane-bound high-affinity receptor expressed on monocytes, macrophages, and eosinophils. Although the expression level of neutrophil CD64 (nCD64) is low in healthy people, ${ }^{1}$ during infections it is upregulated by cytokines, such as interferon- $\gamma($ IFN- $\gamma)$, granulocyte colony-stimulating factor (G-CSF), and lipopolysaccharide (LPS). ${ }^{2,3}$ It plays a role in phagocytosis of targets opsonized with IgG, antibodydependent cytotoxicity, and clearance of immune complexes. ${ }^{4}$ The upregulation of CD64 on neutrophils occurs within 4-6h of neutrophil contact with pro-inflammatory cytokines.

nCD64 would seem to be useful as a diagnostic marker of early phase infection. Indeed, recent studies have shown that nCD64 is a sensitive and specific laboratory marker for the diagnosis of bacterial infection and sepsis in adults, children, and neonates. ${ }^{5-8}$ Furthermore, nCD64 has been reported to increase in patients with viral infections and in rheumatoid arthritis patients with fungal and mycobacterial infections, although the dynamic range in viral infection is about half that of bacterial infection. ${ }^{9,10}$ In addition, it has been reported to be a useful tool in the diagnosis of inflammatory bowel diseases. $^{11}$

Human immunodeficiency virus (HIV)-1 infection is a chronic viral infection that destroys CD4 T lymphocytes that normally orchestrate immune systems, which results in progressive immune deficiency. HIV-infected patents frequently have complicated bacterial and/or fungal infections in the process of their progression to acquired immunodeficiency syndrome (AIDS). For patients newly diagnosed with HIV infection, it is necessary to evaluate the stage of the HIV infection and concomitant infections, including opportunistic and sexually transmitted infections. However, it is difficult to

Department of General Internal Medicine, Kyushu University Hospital, Fukuoka, Japan. 
diagnose these concomitant infections because their symptoms are sometimes mild and unclear.

C-reactive protein (CRP) is a well-known inflammatory marker, and a previous study showed that the CRP level of HIV-infected patients with concomitant infections was significantly higher compared with patients without them. ${ }^{12}$ However, with CRP it is not possible to determine the specific concomitant infection because the CRP level increases as a response to nonspecific inflammatory processes.

Few studies have included analysis of nCD64 in HIVinfected patients. A significant positive correlation was found between the progression of HIV infection and the number of CD64-positive polymorphonuclear leukocytes in perinatally HIV-infected children. ${ }^{13}$ Another report showed that Fc $\gamma$ RI expression on both neutrophils and monocytes was significantly higher in CDC class IV than in class III HIV-infected adult patients, independent of concomitant opportunistic infections. ${ }^{14}$ However, no studies have shown the absolute receptor numbers in $\mathrm{HIV}$-infected patients, the nCD64 of patients with concomitant infections, or the kinetics of nCD64 after the initiation of antiretroviral therapy (ART).

Quantitative measurement of nCD64 can be done easily and rapidly (within $2 \mathrm{~h}$ ) in any laboratory with flow cytometry facilities, and the determination of the absolute receptor number makes possible the comparison of the quantitative receptor studies of different laboratories.

The aim of this study was to determine the absolute neutrophil CD64 expression levels before and after the initiation of ART and to determine the utility of nCD64 as an infection and inflammation marker of HIV infection.

\section{Materials and Methods}

\section{Study population}

A single center observational study was done with 96 consecutive HIV-infected adults who visited Kyushu university hospital between June 2012 and April 2014. Two patients were excluded because they were simultaneously treated for ulcerative colitis, which may have affected the nCD64, leaving the data of 94 patients available for analysis.

The participants were divided into ART, ART naive, and acute/early phase groups. The ART group included 62 patients who had received ART for more than 6 months before the start of the study. The median period from the initiation of ART was 50 months (interquartile range [IQR]: 28-107), and $50(80.6 \%)$ of these patients had an HIV RNA level of less than 20 copies. The ART naive group included $24 \mathrm{HIV}$ positive patients who had not yet received ART. The acute/ early phase group included 8 patients who were thought to be in the acute or early phase of HIV infection, defined as detectable HIV-1 RNA in serum or plasma in the setting of a negative HIV-1 antibody test result or having a positive HIV1 antibody test result with a scattered, positive Western blot band, all of which turned positive after 3 months. A nonpatient group of 110 healthy men in their $40 \mathrm{~s}(44.0 \pm 2.7$ years old) with no obvious signs of infection who took a medical examination at our department were recruited to serve as a control group.

Blood samples were obtained at each visit to our hospital during the observation period and when ART was initiated. However, if a patient in the ART group had any sign of an acute infection, nCD64 was examined at the next visit. He- matological laboratory examinations, such as white blood cell count (WBC) and absolute neutrophil count, were also performed when nCD64 was measured. CRP level was measured by latex-enhanced turbidimetric immunoassay with CRP-Latex II SEIKEN X $2^{\circledR}$ reagents (Denka Seiken, Tokyo, Japan). The HIV RNA level was measured using realtime polymerase chain reaction $\left(\mathrm{COBAS}^{\circledR}\right.$ AmpliPrep/ COBAS $^{\circledR}$ TaqMan $^{\circledR}$ HIV-1 Test, v2.0; Roche Diagnostics, Tokyo, Japan). An HIV RNA level less than 20 copies $/ \mathrm{ml}$ was handled as 20 copies/ml in our analysis, and the HIV RNA level was logarithmically transformed. The absolute CD4 and CD8 T lymphocyte count was assessed from whole blood by flow cytometry.

This study was approved by the Kyushu University Ethics Committee and registered as a clinical trial on the University Hospital Medical Information Network (ID: UMIN00002 1930). Written informed consent was obtained from all patients before enrolment.

\section{Quantitative measurement of neutrophil CD64 expression}

Blood samples used to measure nCD64 were unused portions of the samples obtained for routine blood tests: they were not collected specifically for this study. Flow cytometric analysis was done within $24 \mathrm{~h}$ after blood sampling. Fifty microliters of whole blood was mixed with QuantiBRITE CD64PE/CD45 PerCP (Becton-Dickinson, San Jose, CA) and incubated for $60 \mathrm{~min}$ in the dark at room temperature. The nCD64 was examined using the flow cytometry function of the automated hematology analyzer CELL-DYN Sapphire (Abbott, IL). Lymphocytes, monocytes, and neutrophils were identified and gated by evaluating the CD45/side scattering profiles. A calibration curve was constructed using QuantiBRITE PE beads. The QuantiBRITE PE beads contain four different beads with known numbers of phycoerythrin (PE) molecules that allow the creation of a standard curve for determining the mean number of PE molecules present on a cell. Because the CD64-PE antibody has been designed to bind one PE molecule per antibody, the mean number of CD64 molecules expressed on cells can be calculated using a PE Fluorescence Quantification Kit with QuantiBRITE PE beads. The nCD64 count and the fluorescence intensity of the flow cytometry were stable with a coefficient of variability of less than $2.6 \%$.

\section{Statistical analysis}

All data were analyzed using JMP statistical software (SAS Institute, Cary, NC). Continuous variables are presented as mean value \pm standard deviation or median (firstthird quartiles) and compared using Student's $t$ tests or Mann-Whitney U tests. Group comparisons were carried out using the Tukey-Kramer test or Steel-Dwass nonparametric test. Categorical variables are reported as frequencies and percentages and compared using the Fisher's Exact or Chisquare test. Correlation was evaluated by calculating the Spearman's rank correlation coefficient. Receiver operating characteristic (ROC) curves were constructed and analyzed for the area under the curve (AUC). The optimal cutoff value was calculated using the Youden Index. Statistical tests for comparison of the AUCs were conducted by the nonparametric approach. ${ }^{15}$ Comparison of paired groups was performed by 
Table 1. Baseline Characteristics and Group Comparisons

\begin{tabular}{|c|c|c|c|c|c|c|}
\hline & $A$ & $B$ & $C$ & & $\mathrm{p}$-value & \\
\hline & $A R T(\mathrm{n}=62)$ & ART naive $(\mathrm{n}=24)$ & Acutelearly phase $(\mathrm{n}=8)$ & $A$ versus $B$ & $B$ versus $C$ & $A$ versus $C$ \\
\hline Age, years & $43.5 \pm 12.4$ & $39.8 \pm 9.2$ & $33.4 \pm 7.1$ & n.s. & n.s. & n.s. \\
\hline Male & 58 (93.6) & $23(95.8)$ & $8(100.0)$ & - & - & - \\
\hline $\mathrm{CD} 4, / \mu \mathrm{l}$ & $493 \pm 239$ & $259 \pm 201$ & $367 \pm 148$ & 0.001 & n.s. & n.s. \\
\hline $\mathrm{CD} 8, / \mu \mathrm{l}$ & $695 \pm 270$ & $801 \pm 535$ & $1,680 \pm 818$ & n.s. & 0.029 & 0.011 \\
\hline $\begin{array}{l}\text { HIV RNA, } \\
\text { Log copies/ml }\end{array}$ & $1.41 \pm 0.28$ & $4.73 \pm 1.14$ & $5.41 \pm 0.31$ & $<0.001$ & n.s. & $<0.001$ \\
\hline $\mathrm{WBC}, / \mu \mathrm{l}$ & $5,756 \pm 1,481$ & $4,926 \pm 1,840$ & $6,161 \pm 1,146$ & n.s. & n.s. & n.s. \\
\hline Neutrophil, $/ \mu \mathrm{l}$ & $3,151 \pm 1,048$ & $2,795 \pm 1,835$ & $2,293 \pm 675$ & n.s. & n.s. & n.s. \\
\hline CRP, mg/dl & $0.13 \pm 0.20$ & $0.47 \pm 0.92$ & $0.27 \pm 0.19$ & n.s. & n.s. & 0.038 \\
\hline
\end{tabular}

Data are presented as $n(\%)$ or as mean \pm standard deviation.

$p$-value was determined by the Tukey-Kramer test or Steel-Dwass nonparametric test.

n.s., not significant.

paired $t$-test or Wilcoxon signed-rank test. A $p<0.05$ was regarded as statistically significant in all analyses.

\section{Results}

\section{Baseline characteristics}

The 94 HIV-infected patients were stratified into three groups as follows: 62 receiving ART, 24 ART naive, and 8 in the acute/early phase. Their baseline characteristics are shown in Table 1. The CD4 T lymphocyte count of the ART naive group was significantly lower compared with the ART group $(p=.001)$. The CD8 T lymphocyte count of the acute/early phase group was significantly higher compared with the ART and ART naive groups ( $p=.011$ and .029 , respectively). The HIV RNA level of the ART group was significantly lower compared with the ART naive and acute/early phase groups (both $p<.001$ ). The acute/early phase group had a significantly higher CRP level than the ART group ( $p=.038$ ). No significant differences in WBC or neutrophil count were found.

\section{Comparison of $n C D 64$}

Figure 1 shows the nCD64 of each group. The median (IQR) nCD64 was 1,430 molecules/cell $(979-1,965)$ in the ART group, 2,994 (1,892-3,771) in the ART naive group, $4,625(3,166-5,974)$ in the acute/early phase group, and $1,196(929-1,489)$ in healthy control group. A significant difference in nCD64 was found between the healthy control and ART groups $(p=.041)$. The nCD64 of the ART naive and acute/early phase groups was significantly higher compared with the ART group (both $p<.001$ ). No significant difference in nCD64 was found between the ART naive and acute/early phase groups $(p=.213)$.

\section{Neutrophil CD64 expression in the ART naive group}

Of the 24 patients in the ART naive group, 8 suffered from AIDS-defining diseases and 4 had other infections that required oral or parenteral antimicrobial agents. Table 2 shows the characteristics of these 12 patients. All had nCD64 measured on their first hospital visit. There was no trend in the nCD64 level of patients with specific AIDS-defining disease or other infections.
Table 3 shows a comparison of nCD64 between HIV-infected patients with other infections, including AIDS-defining diseases, and those without other infections. The nCD64 and HIV RNA levels were significantly higher for patients with than without other infections ( $p=.004$ and $<.001$, respectively), although no significant difference in the CRP level was found between these patient groups $(p=.839)$. The CD4 and CD8 T lymphocyte counts were significantly lower for patients with than without other infections ( $p=.005$ and .004 , respectively). The nCD64 count was correlated with the CD4 and CD8 T lymphocyte counts $(\rho=-0.453, p=.026$ and $\rho=-0.424, p=.039)$ and with the HIV RNA level $(\rho=0.587, p=.003)$ (Fig. 2a-c).

ROC analysis was done for nCD64 and CRP to examine their usefulness in diagnosing other infections (Fig. 3). The AUC value of nCD64 was 0.840 (95\% confidence interval (CI): $0.588-0.951)$ and that of CRP was 0.524 (95\% CI: $0.291-0.747)$. The AUC value for nCD64 was significantly higher than that for CRP $(p=.004)$. The use of a cutoff nCD64 value of 3,128 molecules/cell yielded both sensitivity and positivity of $83.3 \%$, with the positive and negative predictive values both at $83.3 \%$.

The patients of the ART naive group had ART initiated after the treatment of concomitant infectious diseases. The

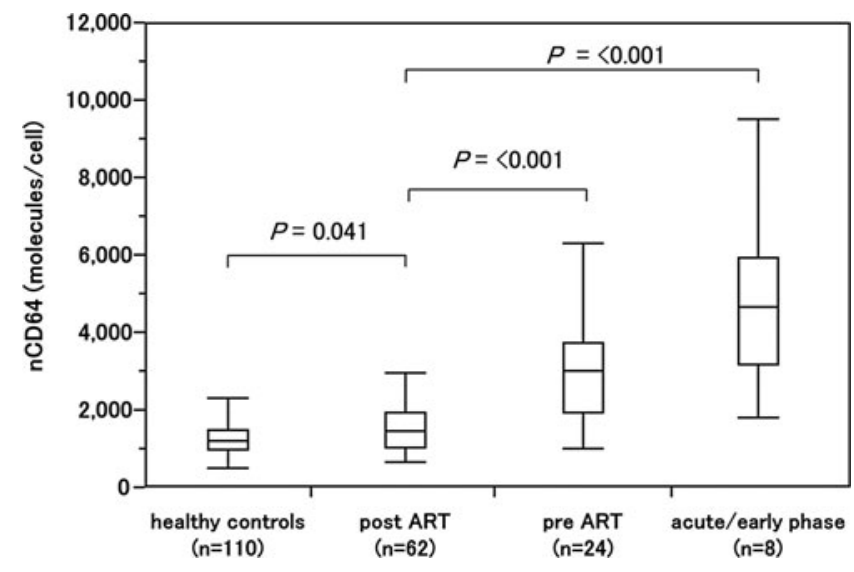

FIG. 1. Between group comparisons of nCD64. Lines inside the boxes show the median values. Boxes indicate the 25th and 75th percentiles, and bars indicate 10th and 90th percentiles. 
Table 2. Characteristics of 12 Patients with AIDS-Defining or Other Infectious Diseases

\begin{tabular}{|c|c|c|c|c|c|c|c|c|c|c|c|}
\hline & Age & $\operatorname{sex}$ & $\begin{array}{l}\text { AIDS-defining } \\
\text { disease }\end{array}$ & $\begin{array}{c}\text { Other } \\
\text { infection }\end{array}$ & $\begin{array}{c}n \mathrm{nD} 64 \\
\text { (molecules/ } \\
\text { cell) }\end{array}$ & $\begin{array}{c}C R P \\
(m g / d l)\end{array}$ & $\begin{array}{l}C D 4 \\
(/ \mu l)\end{array}$ & $\begin{array}{l}C D 8 \\
(/ \mu l)\end{array}$ & $\begin{array}{l}\text { HIVRNA } \\
\quad(\log \\
\text { copies/ } \\
\quad \text { ml) }\end{array}$ & $\begin{array}{cc}W B C & N e u t \\
(/ \mu l) & (/ \mu l)\end{array}$ & $\begin{array}{c}n C D 64 \\
\text { at the } \\
\text { initiation } \\
\text { of ART } \\
\text { (molecules/ } \\
\text { cell) }\end{array}$ \\
\hline 1 & 44 & M & $\begin{array}{l}\text { Pneumocystis pneumonia } \\
\text { Kaposi's sarcoma } \\
\text { Esophageal candidiasis }\end{array}$ & - & 6,316 & 1.55 & 25 & 198 & 6.28 & $6,3405,738$ & 4,141 \\
\hline 2 & 36 & M & $\begin{array}{l}\text { Disseminated NTM } \\
\text { infection, CMV colitis }\end{array}$ & - & 6,187 & 0.43 & 11 & 244 & 7.00 & $4,9104,340$ & 4,434 \\
\hline 3 & 42 & $\mathrm{M}$ & Pneumocystis pneumonia & - & 3,945 & 3.87 & 58 & 506 & 5.45 & $3,7402,248$ & 5,786 \\
\hline 4 & 49 & $\mathrm{~F}$ & Esophageal candidiasis & - & 3,799 & 0.19 & 31 & 249 & 6.72 & $4,5602,590$ & 3,512 \\
\hline 5 & 43 & M & $\begin{array}{l}\text { Pneumocystis pneumonia } \\
\text { CMV retinitis }\end{array}$ & - & 3,688 & 0.11 & 20 & 17 & 6.64 & $1,440 \quad 914$ & - \\
\hline 6 & 36 & M & $\begin{array}{l}\text { Toxoplasmic encephalitis } \\
\text { Pneumocystis pneumonia } \\
\text { Kaposi's sarcoma } \\
\text { Esophageal candidiasis }\end{array}$ & Amebic colitis & 3,387 & 2.54 & 35 & 936 & 5.18 & $5,1704,270$ & 2,836 \\
\hline 7 & 50 & M & Esophageal candidiasis & - & 3,204 & 0.08 & 46 & 1,089 & 5.51 & $3,9301,941$ & - \\
\hline 8 & 57 & M & $\begin{array}{l}\text { Pneumocystis pneumonia } \\
\text { Esophageal candidiasis }\end{array}$ & - & 3,128 & 0.07 & 9 & 114 & 5.94 & $2,6001,768$ & 4,141 \\
\hline 9 & 52 & M & $\begin{array}{lll}2 & -\end{array}$ & $\begin{array}{l}\text { chronic } \\
\text { hepatitis B }\end{array}$ & 5,982 & 0.03 & 350 & 525 & 4.30 & $5,4001,053$ & - \\
\hline 10 & 42 & M & - & Amebic colitis & 3,878 & 0.06 & 518 & 587 & 4.51 & $5,4302,661$ & - \\
\hline 11 & 24 & M & - & syphilis & 2,434 & 0.03 & 209 & 617 & 3.92 & $1,450 \quad 622$ & 2,496 \\
\hline 12 & 42 & M & - & Giardiasis & 1,358 & 0.02 & 413 & 997 & 4.52 & $6,2203,465$ & 969 \\
\hline
\end{tabular}

M, male; F, female; CMV, cytomegalovirus; NTM, nontuberculous mycobacteria.

nCD64 of some patients had increased from the baseline level at the time of the initiation of ART, but for others it decreased (Table 2).

\section{Change of $n C D 64$ of patients receiving $A R T$}

A second analysis was done of the change of nCD64 of patients for whom ART was newly initiated during the study period (new ART group) compared with that of the original ART group. Twenty-two were placed in a newly initiated ART group and 58 in a continuing ART group. These groups did not include patients from the first phase of the study who were lost to follow-up; 10 who did not start ART or had fewer than 12 weeks ART during the study period and 4 who did not have nCD64 measured at 24 weeks. The new ART group included 15 patients of the ART naive group and 7 from the acute/early phase group. nCD64 was measured for 21 patients of the new ART group at week 12 and for 17 at week 24 after the initiation of ART; these data were not available for five patients who did not report at these time points. Of the 22 patients in the new ART group, 14 were prescribed tenofovir disoproxil fumarate (TDF)/emtricitabine (FTC) + raltegravir (RAL), $6 \mathrm{TDF} / \mathrm{FTC} /$ elvitegravir/cobicistat, and 2 abacavir sulfate $(\mathrm{ABC}) /$ lamivudine (3TC) + RAL .

To evaluate the effect of ART on nCD64, the change of nCD64 was compared between the level at the initiation of ART and 24 weeks later in the new ART group and between the first measurement and 24 weeks later in the ART group (Table 4). A significant reduction of the nCD64 during the 24 weeks of treatment was observed in the new ART group, but

Table 3. Comparison of the Characteristics of HiV-Infected Patients With and Without Other INFECTIONS, INCLUding AIDS-DEFINING DiseASES

\begin{tabular}{lcrr}
\hline & With infection $\mathrm{n}=12$ & Without infection $\mathrm{n}=12$ & $\mathrm{p}$-value \\
\hline Age, years & $43.1 \pm 8.7$ & $36.6 \pm 9.0$ & .085 \\
Male & $11(91.7)$ & $12(100.0)$ & 1.000 \\
nCD64, molecules/cell & $3,942 \pm 1,519$ & $2,300 \pm 784$ & .004 \\
CRP, mg/dl & $0.75 \pm 1.26$ & $0.20 \pm 0.19$ & .839 \\
CD4, $/ \mu \mathrm{l}$ & $144 \pm 182$ & $375 \pm 150$ & .005 \\
$\mathrm{CD} 8, / \mu \mathrm{l}$ & $507 \pm 358$ & $1,095 \pm 530$ & .004 \\
$\mathrm{HIV} \mathrm{RNA}$, Log copies/ml & $5.50 \pm 1.04$ & $3.96 \pm 0.57$ & $<.001$ \\
WBC $/ \mu \mathrm{l}$ & $4,266 \pm 1,683$ & $5,586 \pm 1,815$ & .078 \\
Neutrophil $/ \mu \mathrm{l}$ & $2,634 \pm 1,561$ & $2,955 \pm 1,231$ & .582 \\
\hline
\end{tabular}

Data are presented as $n(\%)$ or as mean \pm standard deviation. 
FIG. 2. Correlation of nCD64 with CD4, CD8, and HIV RNA level. (a) Correlation of nCD64 with CD4 lymphocyte count. (b) Correlymphocyte count. (c) Correlation of nCD64 with HIV RNA level. lation of nCD64 with $\mathrm{CD} 8$
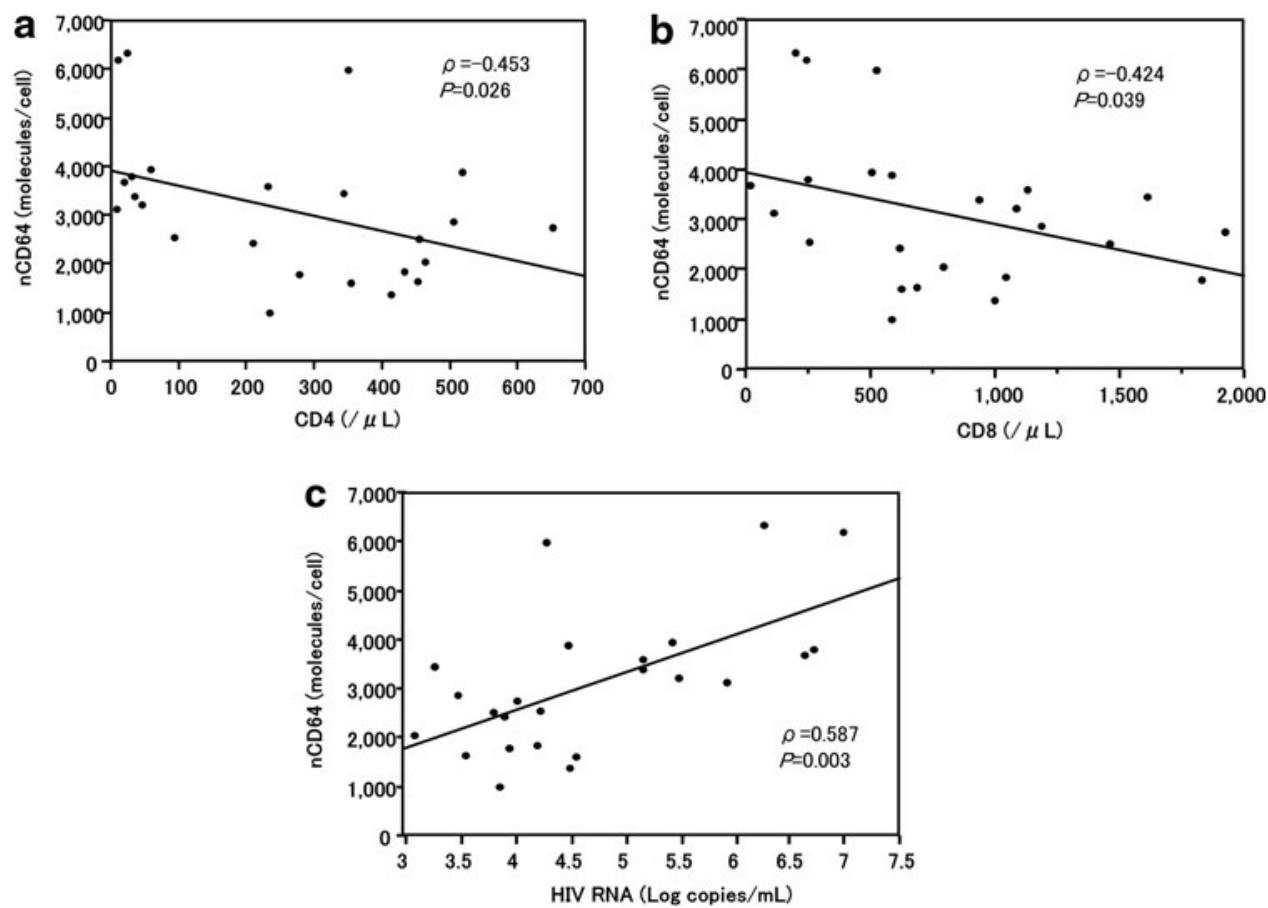

not in the ART group. Although there were significant increases in the CD4 T lymphocyte, WBC, and neutrophil counts and a significant reduction of the HIV RNA level in the new ART group, no significant change of the parameters was observed during the 24 weeks in the ART group.

\section{Change of $n C D 64$ in immune reconstitution inflammatory syndrome}

In the new ART group, two patients experienced immune reconstitution inflammatory syndrome (IRIS) after ART was initiated. One had paradoxical pneumocystis pneumonia (PCP)-IRIS and unmasking progressive multifocal leukoencephalopathy (PML)-IRIS. The clinical course of this 42-year-old patient diagnosed with AIDS and PCP is shown in Figure 4. ART (ABC/3TC + RAL) was initiated after the treatment of PCP for 21 days, and PCP-IRIS occurred 8 days after initiating ART. At that time, a remarkable elevation of both nCD64 and the CRP level was observed. About 7 weeks later, he suffered from a memory disorder and was diagnosed with unmasking PML-IRIS. Furthermore, 22 weeks later he suffered from an exacerbation of herpes zoster. Elevation of the nCD64, but not the CRP level, was observed at each testing point. Thirty-five weeks later, he had swollen tonsils and cervical lymphadenopathy with a remarkable elevation of nCD64. Although antimicrobial agents were administered for the tonsillitis, his symptoms were not improved, and subsequently, he was diagnosed with AIDS related diffuse large B-cell lymphoma.

The other was a 36-year-old patient who experienced paradoxical IRIS from disseminated nontuberculous mycobacteria (NTM) infection. The clinical course of this patient is shown in Figure 5. His diagnosis included AIDS, disseminated NTM infection, and cytomegalovirus (CMV) colitis. ART (TDF/FTC + RAL) was initiated after 4 weeks treatment for CMV colitis and disseminated NTM infection. The nCD64 remarkably elevated beyond 20,000 molecules/cell after the initiation of
ART, and a high fever persisted. New skin lesions from the NTM infection developed 7 weeks later, and the treatment for the NTM infection was strengthened with the continuation of ART. Although simultaneous changes of nCD64 and CRP were observed for these patients, the fluctuation range of nCD64 was greater compared with CRP.

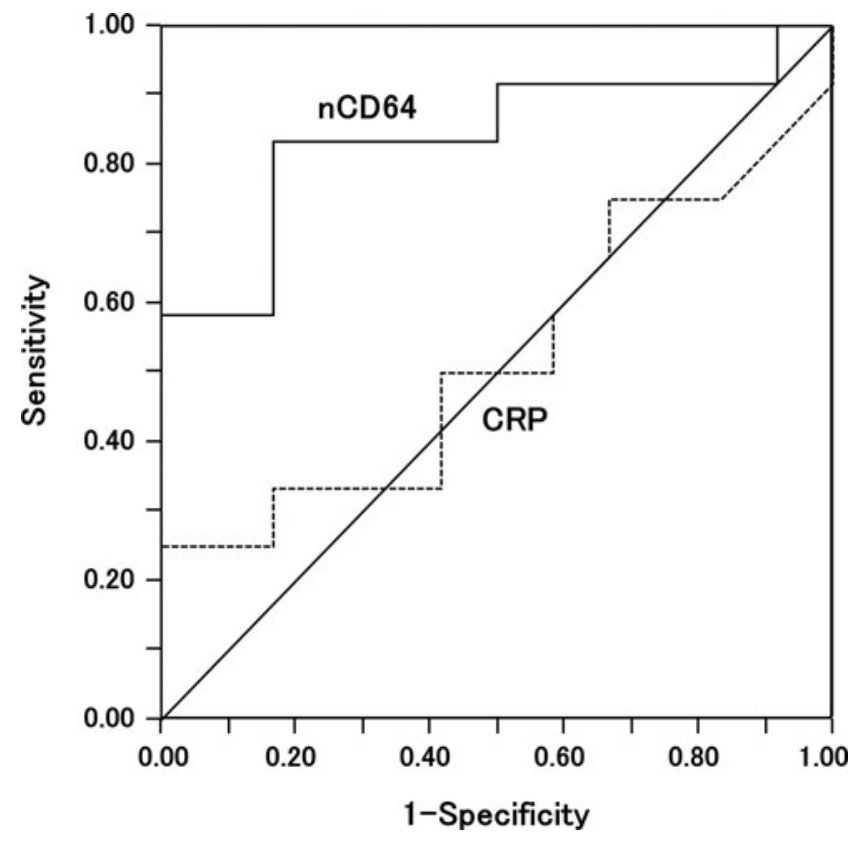

FIG. 3. ROC analysis of the utility of nCD64 and CRP for the diagnosis of concomitant infections. The AUC value of nCD64 was 0.840 (95\% CI: 0.588-0.951) and that of CRP was 0.524 (95\% CI: 0.291-0.747). ROC, receiving operating characteristic; AUC, area under the curve; CI, confidence interval. 
Table 4. nCD64 and Other HIV Infection Marker Level Change at Baseline and 24 Weeks

\begin{tabular}{|c|c|c|c|c|c|c|}
\hline & \multicolumn{3}{|c|}{$A R T$ group $(\mathrm{n}=58)$} & \multicolumn{3}{|c|}{ New ART group $(\mathrm{n}=17)$} \\
\hline & $\begin{array}{c}\text { Week } 0 \\
\text { (first measurement) }\end{array}$ & Week 24 & p-value & $\begin{array}{c}\text { Week } 0 \\
\text { (initiation of ART) }\end{array}$ & Week 24 & $\mathrm{p}$-value \\
\hline nCD64, molecules/cell & $1,615 \pm 894$ & $1,608 \pm 746$ & .402 & $3,337 \pm 1,825$ & $2,067 \pm 810$ & .004 \\
\hline $\mathrm{CD} 4, / \mu \mathrm{l}$ & $484 \pm 256$ & $502 \pm 240$ & .280 & $289 \pm 177$ & $517 \pm 233$ & $<.001$ \\
\hline $\mathrm{CD} 8, / \mu \mathrm{l}$ & $716 \pm 295$ & $734 \pm 314$ & .488 & $997 \pm 489$ & $982 \pm 388$ & .885 \\
\hline HIV RNA, Log copies/ml & $1.42 \pm 0.29$ & $1.34 \pm 0.15$ & .020 & $4.86 \pm 0.69$ & $1.45 \pm 0.21$ & $<.001$ \\
\hline $\mathrm{WBC}, / \mu \mathrm{l}$ & $5,788 \pm 1,861$ & $5,787 \pm 1,766$ & .879 & $4,703 \pm 2,185$ & $5,717 \pm 1,638$ & .013 \\
\hline Neutrophil, $/ \mu 1$ & $3,207 \pm 1,524$ & $3,077 \pm 1,144$ & .493 & $2,171 \pm 1,332$ & $2,756 \pm 1,095$ & .047 \\
\hline CRP, mg/dl & $0.13 \pm 0.20$ & $0.15 \pm 0.30$ & .448 & $0.19 \pm 0.35$ & $0.08 \pm 0.08$ & .256 \\
\hline
\end{tabular}

Data are expressed as mean \pm standard deviation.

\section{Change of nCD64 after the initiation of $A R T$}

Figure 6 shows the change of nCD64 during the 24 weeks after the initiation of ART in the new ART group. The two patients who had experienced IRIS were excluded. An upward trend in nCD64 was observed at weeks 2 and 4 after the initiation of ART, followed by a significant reduction at weeks 12 and 24. At the time of nCD64 elevation after the initiation of ART, no symptoms were seen.

The median difference in nCD64 at baseline (week 0: at ART initiation) and the maximum within the first 12 weeks of ART ( $\triangle$ nCD64 within 12 week) was 862 molecules/cell (IQR: 261-2,605). These 20 patients were divided into a group whose $\angle \mathrm{nCD} 64$ within the first 12 weeks was higher than or equal to 1,000 molecules/cell $(n=8)$ (high $\angle$ nCD64 group) and another lower than 1,000 molecules/cell $(n=12)$ (low $\angle$ nCD64 group). Table 5 shows the characteristics of these subgroups. The high $\triangle \mathrm{nCD} 64$ group had a significantly higher HIV RNA level at the initiation of ART and a higher decline of HIV RNA level at 12 weeks compared with those of the low $\triangle \mathrm{nCD} 64$ group ( $p=.011$ and .049 , respectively). Although no significant difference in the CD4 T lymphocyte count was found between these two subgroups, the high $\angle \mathrm{nCD} 64$ group tended to have a lower CD4 T lymphocyte count at baseline $(p=.070)$. No significant between group differences were found in the CD8 T lymphocyte count or change in the CD4 and CD8 T lymphocyte counts after the initiation of ART.

\section{Discussion}

To our knowledge, the present study is the only one to evaluate the absolute neutrophil CD64 expression levels of patients with HIV infection before and after the initiation of ART and to show the role of nCD64 as an infection and inflammation marker of HIV infection. The present study shows that HIV status is significantly associated with nCD64. The nCD64 was highest in the acute/early phase of HIV infection and decreased in the chronic phase. In addition, the nCD64 of HIV-infected patients receiving ART was significantly lower compared with those who were ART naive, and it did not reduce to healthy control levels despite virus suppression by ART. Furthermore, we showed that the nCD64 of the ART naive group was positively correlated with the HIV RNA level and negatively correlated with the CD4 and CD8 T lymphocyte counts, indicating that nCD64 increases with the progression of HIV infection, similar to previous reports. ${ }^{13,14}$ These data suggest that nCD64 would be a useful inflammation marker because it reflects the stage of HIV infection.

The increase in nCD64 with the progression of HIV infection may be the result of chronic inflammation induced by factors, including HIV proteins, inflammatory cytokines such as IFN- $\gamma$, and microbial translocation. Previous studies have shown that the IFN- $\gamma$ concentration in sera is correlated with the stage of HIV infection. ${ }^{14,16}$ In addition, HIV-infected

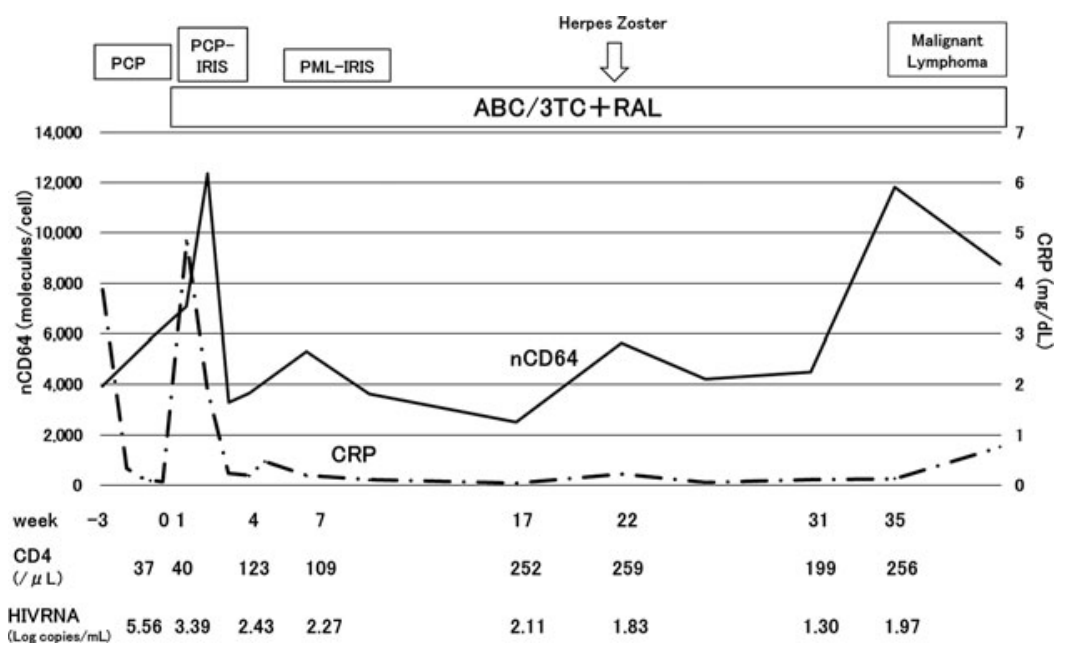

FIG. 4. Clinical course of a patient with paradoxical pneumocystis pneumonia and unmasking progressive multifocal leukoencephalopathy immune reconstitution inflammatory syndrome. PCP, pneumocystis pneumonia; IRIS, immune reconstitution inflammatory syndrome; PML, progressive multifocal leukoencephalopathy; ABC, abacavir sulfate; 3TC, lamivudine; RAL, raltegravir; nCD64, neutrophil CD64 expression level. 
FIG. 5. Clinical course of a patient with paradoxical immune reconstitution inflammatory syndrome by disseminated nontuberculous mycobacterium infection. nCD64, neutrophil CD64 expression level; NTM, nontuberculous mycobacterium; LVFX, levofloxacin; SM, streptomycin; AMK, amikacin; RBT, rifabutin; EB, ethambutol; CAM, clarithromycin; AZM, azithromycin; TDF, tenofovir disoproxil fumarate; FTC, emtricitabine; RAL, raltegravir; DTG, dolutegravir.

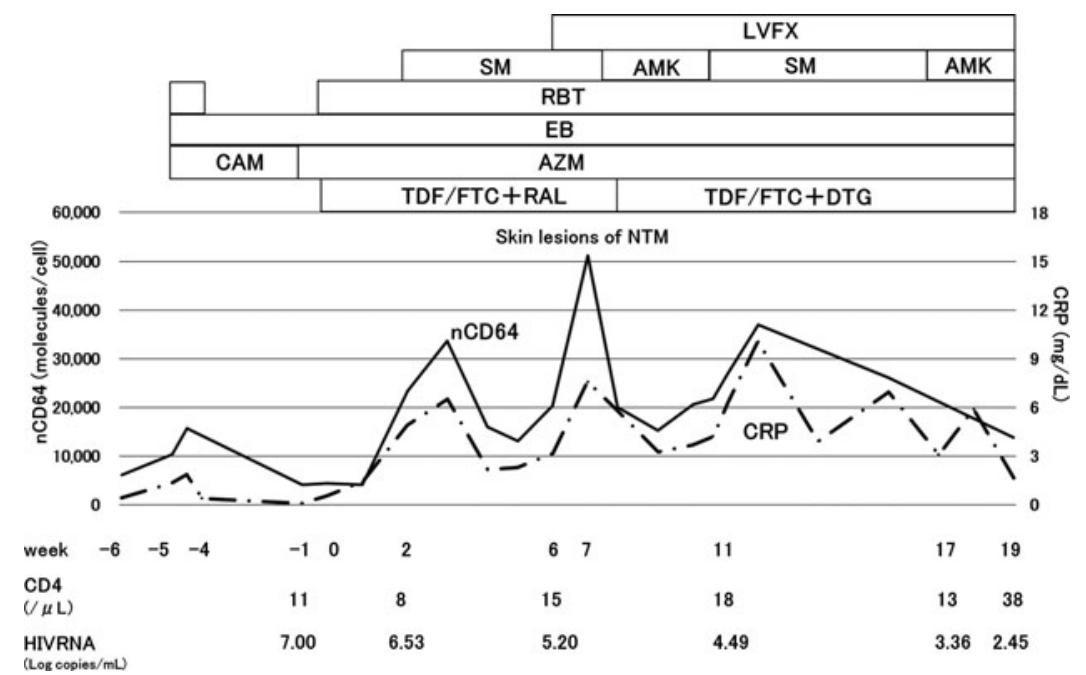

patients have significantly higher plasma LPS levels than uninfected patients, and the level decreases with ART. LPS reflects microbial translocation in $\mathrm{HIV}$-infected patients ${ }^{17}$ that is associated with HIV progression. ${ }^{18}$

In the present study, there was a significant difference in the nCD64 of our healthy control and ART groups, even if their HIV RNA levels were suppressed less than 20 copies. It has been reported that many inflammatory markers, including IFN- $\gamma$, in HIV suppressed patients are at the same levels as HIV-uninfected patients, although $\mathrm{C}-\mathrm{X}-\mathrm{C}$ motif chemokine 10 (CXCL10), which is also known as IFN- $\gamma$-induced protein 10 (IP-10), soluble cytokine receptors (sCD27), soluble TNF receptor 2 (sTNFR2), pro-inflammatory cytokines such as TNF- $\alpha$ and $\mathrm{B}$ cell activating factor that belong to the tumor necrosis factor family (BAFF), soluble scavenger receptor sCD14, and high sensitivity CRP (hsCRP) remain abnormally high among HIV suppressed patients. ${ }^{19}$ Furthermore, the LPS level generally decreases after the initiation of ART,

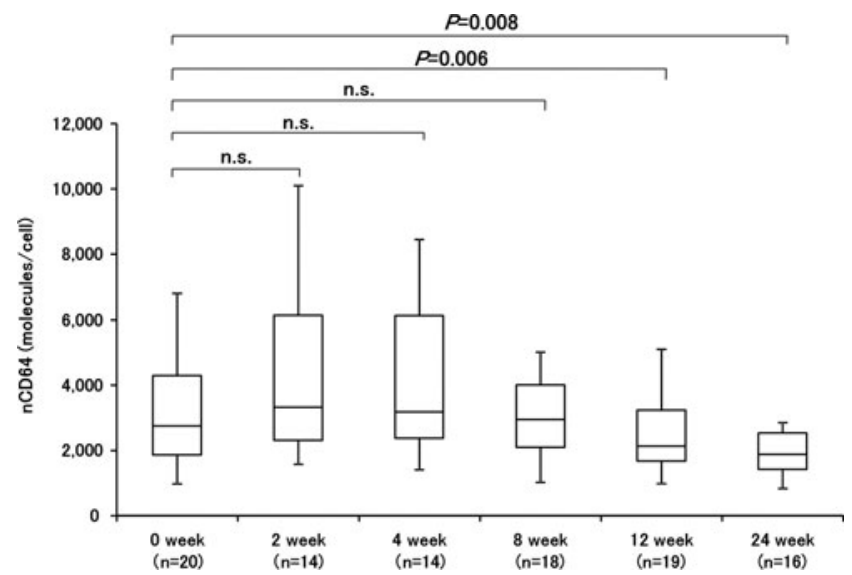

FIG. 6. Neutrophil CD64 expression level of the patients in the new ART group, excepting two patients who experienced IRIS after the initiation of ART. n.s., not significant. Median neutrophil CD64 expression level is indicated by the line inside the box, 25th-75th percentiles are indicated by the boxes, and minimal-maximal values are shown by the vertical bars. nCD64, neutrophil CD64 expression level. but rarely to the same level as that of healthy controls. ${ }^{20,21}$ The higher nCD64 in the continuing ART than that in the healthy control group might be related to residual immune activation and inflammation, such as higher levels of LPS and sCD14, in suppressed HIV patients. A higher plasma LPS level was reported to be a risk factor for HIV-associated dementia, ${ }^{22}$ and LPS and sCD14 were associated with an increase in the yearly rate of change in the carotid-intima thickness of HIV-infected patients. ${ }^{23}$ Therefore, even with HIV suppression by ART, it is important to carefully follow patients whose nCD64 levels are high.

We compared the nCD64 of HIV-infected patients with and without concomitant infections. Although there was no significant difference in the CRP level, nCD64 was significantly higher in patients with concomitant infections. The cutoff nCD64 value for the diagnosis of a concomitant infection was 3,128 molecules/cell with sensitivity and positivity both at $83.3 \%$. However, the higher nCD64 of patients with concomitant infections may have been influenced by the stage of HIV infection, because 8 of 12 patients were suffering from AIDS-defining diseases and there were significant differences between the groups in the HIV RNA level and CD4 and CD8 T lymphocyte counts. Therefore, the utility of nCD64 for judging the presence or absence of the concomitant infections of HIV-infected patients, it is necessary to examine nCD64 in patients with or without infections whose CD4 and HIV RNA level are the same level.

It has been suggested that nCD64 can be used for monitoring the response to sepsis treatment. A previous study reported that patients with sepsis receiving inappropriate antibiotics had persistently elevated nCD64, whereas expression decreased over time in patients receiving appropriate antibiotics. ${ }^{24}$ However, in this study, the nCD64 of some HIV patients with concomitant infections increased during treatment, even though they were treated according to the guidelines and their symptoms were improved. Because we treated concomitant infections before initiating ART, the untreated HIV infection may have influenced nCD64.

We also examined the kinetics of nCD64 after the initiation of ART. Although ART dramatically reduces the HIV viral level, the effect of ART on immune activation and inflammation is unclear. A recent study revealed that many 
Table 5. Comparison of the Characteristics of Patients with $\angle$ NCD64 WAS $\geqq 1,000$ MOLECULES/CELL AND THOSE $<1,000$

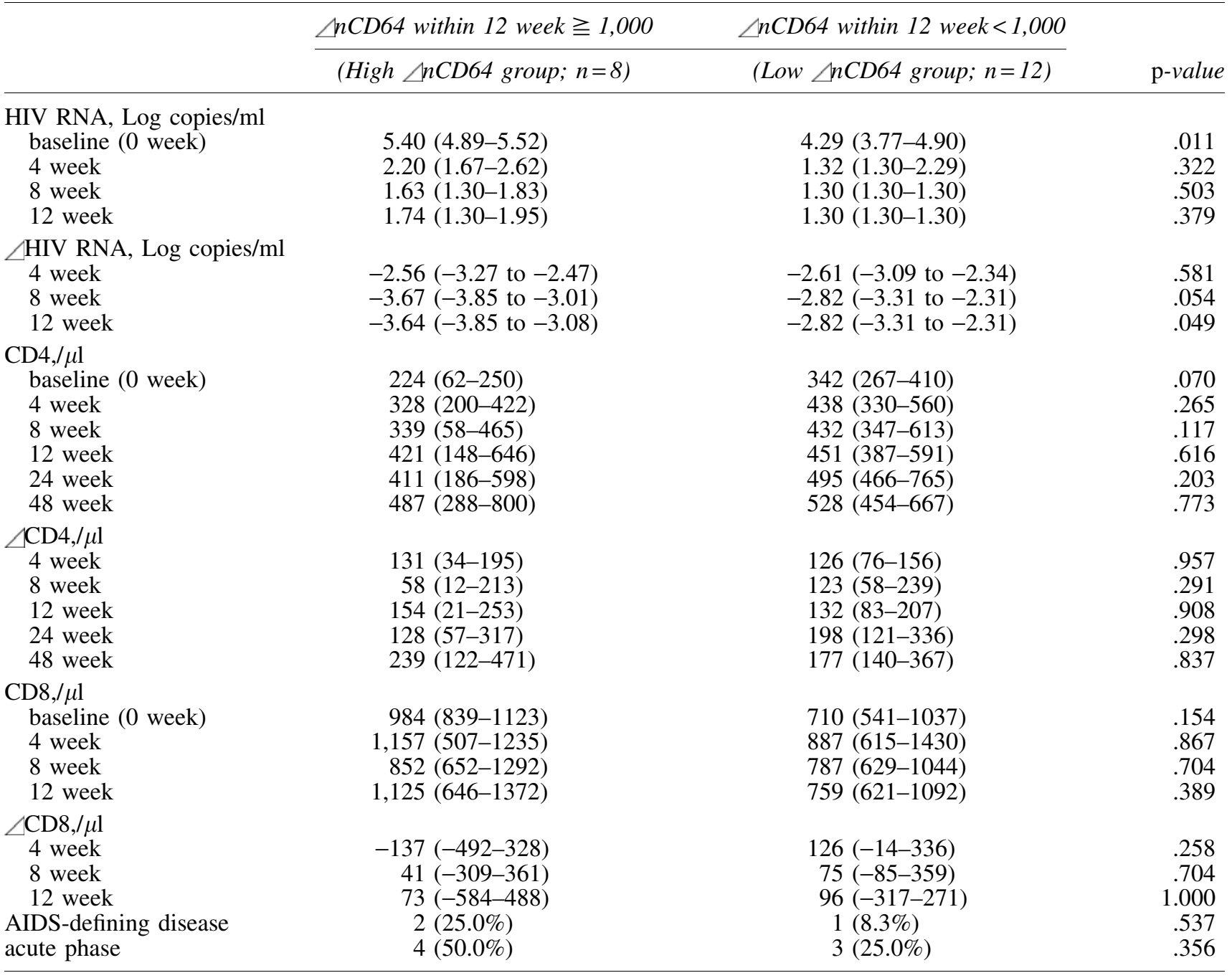

inflammatory biomarkers, including IFN- $\gamma$, CXCL-10, IL-2, IL-10, and TNF- $\alpha$, declined in the first year after the initiation of ART and that the annual change in these markers after the first year was essentially zero. ${ }^{19}$ In the present study, nCD64 was decreased significantly at 24 weeks after the initiation of ART, but did not change after 24 weeks in patients receiving ART. This indicates that the decline of nCD64 during the first 24 weeks on ART reflects the decline of inflammatory cytokines, in addition to that of the HIV RNA level.

In the present study, two patients experienced IRIS during the observation period. In the first case, when paradoxical PCP-IRIS occurred, remarkable elevation of both nCD64 and the CRP level was found. However, when unmasking PMLIRIS occurred, nCD64 was slightly elevated, without elevation of the CRP level. Compared with CRP, the dynamic range of nCD64 is considerably wider, suggesting that nCD64 might be a more sensitive marker for immune activation than CRP when IRIS occurs. In addition, fluctuation of the nCD64 level might vary according to the pathogen responsible for IRIS.

The second case had paradoxical IRIS from disseminated NTM infection. Although the NTM infection was treated for 4 weeks before the start of ART, high fever continued and
nCD64 went over 20,000 molecules/cell after the initiation of ART. The nCD64 in this case was higher than that seen in infection or IRIS by other types of pathogen. There are a few reports that have measured nCD64 for mycobacterium infections, and $\mathrm{nCD} 64$ tends to be induced strongly by mycobacteria. ${ }^{10,25}$ At present, nCD64 cannot be used to discriminate between the IRIS-induced pathogens of HIV-infected patients because of the few cases studied, but mycobacterium-IRIS should be considered in the case of remarkable elevation, over 20,000 molecules/cell, after the initiation of ART.

We observed that the nCD64 of the patients in the new ART group tended to increase temporarily 2-4 weeks after the initiation of ART, without symptoms, then to be significantly decreased at 12 weeks. This phenomenon might reflect immune reconstitution. A greater than 90 percent HIV viral level decline is seen within 1-2 weeks after starting ART, and this decline in viral level usually persists during the next 8-12 weeks, then stabilizes. An increase in immune effector cells occurs in inverse proportion to the fall in the HIV viral level. Both memory CD4 and CD8 T lymphocytes increase rapidly after the initiation of ART. A past study reported that CD8 T lymphocyte increased immediately and continued for the first 6 weeks, with a subsequent decrease to the baseline level, and 
that the initial changes were mainly caused by an initial increase in CD8 memory T lymphocyte count. ${ }^{26}$ The temporary nCD64 elevation might reflect the increase of memory CD8 and CD4 T lymphocytes, although the $\triangle \mathrm{CD} 4$ and $\angle \mathrm{CD} 8 \mathrm{~T}$ lymphocyte counts at each point did not differ between the high and low $\triangle \mathrm{nCD} 64$ groups in this study. The fact that the baseline HIV RNA level and the decline of the HIV viral level after starting ART were higher in the high than in the low $\triangle \mathrm{nCD} 64$ group and the tendency for the high $\triangle \mathrm{nCD} 64$ group to have a lower baseline CD4 T lymphocyte count supports this hypothesis.

We have some limitations in this study. First, the number of patients was small and we could not conclude the utility of nCD64 for judging the presence or absence of the concomitant infection. Second, we did not compare the clinical characteristics of the HIV-infected patients and healthy controls because, in another of our studies involving non$\mathrm{HIV}$-infected persons, we found no significant associations between nCD64 and smoking, body mass index, or comorbidities such as diabetes mellitus or hypertension. Only age was significantly associated (data not shown). Therefore, we feel that differences in the clinical characteristics of the groups would have had little effect on the nCD64 results. Furthermore, we were unable to find any studies about the association between nCD64 and medication. However, we considered the anti-inflammatory action of steroids and excluded persons who were taking them from the healthy control group. None of the HIV-infected patients were taking steroids at the time of nCD64 measurement. Third, although we excluded patients who had self-reported having suffered from infectious diseases, we did not directly examine them. This leaves the possibility that some persons with infectious diseases were included in the healthy control group. Fourth, 10 HIV-infected patients did not start ART or have a sufficient time receiving it to enroll the second prospective part of the study; 32 patients had not received ART in the first crosssectional part of the study. Furthermore, not all of the enrolled patients had nCD64 measured at 12 or 24 weeks after starting ART. The low follow-up rate might act as selection bias. Because this is a preliminary study about the nCD64 of HIVinfected patients, future large-scale study will be necessary.

In conclusion, nCD64 would be useful as a marker of systemic inflammation associated with HIV infection. More clinical experience will be necessary to clarify the utility of nCD64 and studies of its association with other biomolecular markers are necessary.

\section{Acknowledgments}

The authors are grateful to Drs. Haru Mukae, Takeshi Ihara, Takeo Hayashi, Rinne Takeda, Yoshifumi Kato, Yuuki Tanaka, Sho Yamasaki, and Masaru Sakiyama from the Department of General Internal Medicine, Kyushu University Hospital for their assistance with data collection for this study.

\section{Author Disclosure Statement}

Norihiro Furusyo has received grants from Taisho Toyama Pharmaceutical Co., Ltd., Daiichi Sankyo Co., Ltd., Takeda Pharmaceutical Company Limited, Mitsubishi Tanabe Pharma Corporation, Chugai Pharmaceutical Co., Ltd., Janssen Pharmaceutical K.K., Bristol-Myers Squibb, and
AbbVie Inc. The other authors declare that they have no conflicts of interest. This study received no specific funding.

\section{References}

1. Elghetany MT: Surface antigen changes during normal neutrophilic development: A critical review. Blood Cells Mol Dis 2002;28:260-274.

2. van de Winkel JG, Anderson CL: Biology of human immunoglobulin G Fc receptors. J Leukoc Biol 1991;49:511524.

3. Wagner C, Deppisch R, Denefleh B, Hug F, Andrassy K, Hansch GM: Expression patterns of the lipopolysaccharide receptor $\mathrm{CD} 14$, and the FCgamma receptors CD16 and CD64 on polymorphonuclear neutrophils: Data from patients with severe bacterial infections and lipopolysaccharideexposed cells. Shock 2003;19:5-12.

4. Schiff DE, Rae J, Martin TR, Davis BH, Curnutte JT: Increased phagocyte Fc gammaRI expression and improved Fc gamma-receptor-mediated phagocytosis after in vivo recombinant human interferon-gamma treatment of normal human subjects. Blood 1997;90:3187-3194.

5. Wang X, Li ZY, Zeng L, et al.: Neutrophil CD64 expression as a diagnostic marker for sepsis in adult patients: A meta-analysis. Crit Care 2015;19:245.

6. Li S, Huang X, Chen Z, et al.: Neutrophil CD64 expression as a biomarker in the early diagnosis of bacterial infection: A meta-analysis. Int J Infect Dis 2013;17:e12-e23.

7. Cid J, Aguinaco R, Sanchez R, Garcia-Pardo G, Llorente A: Neutrophil CD64 expression as marker of bacterial infection: A systematic review and meta-analysis. J Infect 2010; 60:313-319.

8. Jia LQ, Shen YC, Hu QJ, et al.: Diagnostic accuracy of neutrophil CD64 expression in neonatal infection: A metaanalysis. J Int Med Res 2013;41:934-943.

9. Nuutila J, Hohenthal U, Laitinen I, et al.: Simultaneous quantitative analysis of FcgammaRI (CD64) expression on neutrophils and monocytes: A new, improved way to detect infections. J Immunol Methods 2007;328:189-200.

10. Matsui T, Ohsumi K, Ozawa N, et al.: CD64 on neutrophils is a sensitive and specific marker for detection of infection in patients with rheumatoid arthritis. J Rheumatol 2006;33: 2416-2424.

11. Tillinger W, Jilch R, Jilma B, et al.: Expression of the highaffinity IgG receptor FcRI (CD64) in patients with inflammatory bowel disease: A new biomarker for gastroenterologic diagnostics. Am J Gastroenterol 2009;104:102-109.

12. Noursadeghi M, Miller RF: Clinical value of C-reactive protein measurements in HIV-positive patients. Int J STD AIDS 2005;16:438-441.

13. Moallem HJ, Kalayci O, Homel $\mathrm{P}$, et al: Expression of $\mathrm{Fc}$ (gamma)r1 (CD64) on polymorphonuclear leucocytes during progression to acquired immunodeficiency syndrome in perinatally human immunodeficiency virusinfected children. Scand J Immunol 2000;52:184-189.

14. Capsoni F, Minonzio F, Ongari AM, et al: Increased expression of IgG Fc receptor type I on neutrophils and monocytes from HIV-infected subjects. Clin Exp Immunol 1992;90: 175-180.

15. DeLong ER, DeLong DM, Clarke-Pearson DL: Comparing the areas under two or more correlated receiver operating characteristic curves: A nonparametric approach. Biometrics 1988;44:837-845.

16. Fuchs D, Hausen A, Reibnegger G, et al:: Interferongamma concentrations are increased in sera from 
individuals infected with human immunodeficiency virus type 1. J Acquir Immune Defic Syndr 1989;2:158-162.

17. Brenchley JM, Price DA, Schacker TW, et al.: Microbial translocation is a cause of systemic immune activation in chronic HIV infection. Nat Med 2006;12:1365-1371.

18. Nowroozalizadeh S, Mansson F, da Silva Z, et al.: Microbial translocation correlates with the severity of both HIV-1 and HIV-2 infections. J Infect Dis 2010;201:1150-1154.

19. Wada NI, Jacobson LP, Margolick JB, et al.: The effect of HAART-induced HIV suppression on circulating markers of inflammation and immune activation. Aids 2015;29: 463-471.

20. Jiang W, Lederman MM, Hunt $\mathrm{P}$, et al:: Plasma levels of bacterial DNA correlate with immune activation and the magnitude of immune restoration in persons with antiretroviral-treated HIV infection. J Infect Dis 2009;199: 1177-1185.

21. Baroncelli S, Galluzzo CM, Pirillo MF, et al.: Microbial translocation is associated with residual viral replication in HAART-treated HIV+ subjects with <50copies/ml HIV-1 RNA. J Clin Virol 2009;46:367-370.

22. Ancuta $\mathrm{P}$, Kamat A, Kunstman KJ, et al.: Microbial translocation is associated with increased monocyte activation and dementia in AIDS patients. PLoS One 2008; 3:e2516.

23. Kelesidis T, Kendall MA, Yang OO, Hodis HN, Currier JS: Biomarkers of microbial translocation and macrophage activation: Association with progression of subclinical atherosclerosis in HIV-1 infection. J Infect Dis 2012;206: $1558-1567$.

24. Dimoula A, Pradier O, Kassengera Z, Dalcomune D, Turkan H, Vincent JL: Serial determinations of neutrophil CD64 expression for the diagnosis and monitoring of sepsis in critically ill patients. Clin Infect Dis 2014;58:820-829.

25. Aleman M, Beigier-Bompadre M, BorghettiC, et al: Activation of peripheral blood neutrophils from patients with active advanced tuberculosis. Clin Immunol 2001;100:87-95.

26. Notermans DW, Pakker NG, Hamann D, et al.: Immune reconstitution after 2 years of successful potent antiretroviral therapy in previously untreated human immunodeficiency virus type 1-infected adults. J Infect Dis 1999; 180:1050-1056.

Address correspondence to: Norihiro Furusyo Department of General Internal Medicine Kyushu University Hospital 3-1-1 Maidashi, Higashi-ku Fukuoka 812-8582

Japan

E-mail: furusyo@gim.med.kyushu-u.ac.jp 